\title{
IL SACRO NEI NOMI DEI LIQUORI
}

\author{
Silvia Corino Rovano \\ Università di Torino, Italia
}

\section{The sacred in names of liqueurs}

\begin{abstract}
Nothing seems more remote from religion than alcohol, but everyone in Italy knows spirits named San Simone, San Marzano, etc. Moreover, many abbeys used to produce spirits which were believed to be good for health and the drinks often bore the name of the abbey's patron saint. Cocktails may have "holy" names as well.

The paper is aimed at investigating the relationship between tradition and drinking alcohol, a less religious habit, which may be considered a vice.

Keywords: anthroponyms, saints, tradition, spirits, wine.
\end{abstract}

"A San Martin a son fait tuti i vin"1 a san Martino i vini sono pronti per la spillatura. Questo detto piemontese si inserisce in un ampio contesto tradizionale in cui i santi hanno rappresentato non solo un esempio di virtù cristiana ma anche un ruolo più popolare di vicinanza alla vita contadina. Dal calendario, più che dall'alto dei cieli, i santi sembrano osservare e proteggere le fasi significative della vendemmia e del raccolto e più in generale dell'agricoltura, e non sono estranei neanche al pagamento dei tributi; proprio San Martino gioca un ruolo di primo piano nel pagamento degli affitti dei campi (11 novembre, fine del calendario rurale) e nel periodo in cui si fanno i traslochi ${ }^{2}$.

Inoltre il vino, dice il noto proverbio, insieme al tabacco e alla lussuria riducono l'uomo in cenere. Ciononostante alcuni vini o liquori portano il nome di un santo. Questa complessa relazione tra santi e liquori, una contraddizione tra la sacralità del nome e la peccaminosità del contenuto, merita secondo noi un'indagine.

Anche sulle mense regali erano presenti vini che portavano nomi di santi. I sovrani piemontesi, ad esempio, non si facevano mancare vini pregiati e al tempo di Carlo Alberto i vini santi erano stranieri; oltre al Sauterne e al Bordeaux Lafitte, infatti, vengono acquistati il St. Peray, il St. George, il St. Lucar ${ }^{3}$. Oltre a questi portatori di nomi di santi ve ne è uno detto "Monaca".

1 DSA, s.v. Vin.

2 Ricordiamo il fare San Martino come traslocare.

3 ASTo (=Archivio di Stato di Torino), Sezioni Riunite, Real Casa, Azienda Generale della Real Casa, Rego di Carlo Alberto, f. 1482. Gli elenchi presenti nel fascicolo vanno dal 1834 al 1839.

4 Ibidem. 
L'appellativo vicino al sacro può trarre in inganno: costituisce un esempio il "Vin Santo" toscano un vino utilizzato non tanto per celebrare la messa ma come vino da dessert accompagnato da dolci regionali tipici. Il riferimento onomastico di questo vino è legato piuttosto alla conservazione delle uve che veniva protratta fino alla Settimana Santa 5 .

In questo panorama variegato si sono individuati tra i nomi di bevande alcoliche alcune tipicità che consentono di fare dei raggruppamenti descrittivi. È evidente che occorre ripercorrere la storia di ciascun liquore per motivare il legame con il nome santo.

I due raggruppamenti principali sono legati all'origine marchionimica o toponimica o antroponimica dell'elisir.

\section{Denominazione di origine marchionimica}

Il primo gruppo è quello guidato da un marchionimo che porta il nome di un santo ma che non presenta legami con l'alcol. O meglio, la bevanda alcolica porta il nome di un santo in quanto parte di un "marchio santo".

Questo caso emblematico è rappresentato dai prodotti della ditta San Pellegrino. Infatti, la ditta che ormai è un colosso internazionale "ha un suo mondo", come recita il sito, ed ha creato un' intera famiglia di prodotti basati sul nome originario del marchionimo.

Fanno quindi riferimento a questa denominazione tanto bevande analcoliche (aranciate, chinotti, cedrate, ecc.) quanto bevande alcoliche come il "Cocktail San Pellegrino".

In questo caso, in effetti, non vi è alcun rapporto con il santo e la popolarità dei prodotti fa parte del marketing contemporaneo. A testimoniarlo il fortunato bitter basato su una trasformazione del nome, il cui successo è stato sancito da martellanti campagne pubblicitarie: il "Sanbitter". In sintesi il protagonista è il marchionimo e tutti i nomi della ditta lo acquistano sistematicamente come parte del nome in uno schema "x San Pellegrino": "Aranciata San Pellegrino", "Chinotto San Pellegrino" e quindi anche "Cocktail San Pellegrino".

Anche se meno conosciute, altre tipologie di liquori seguono questa logica e il nome del marchionimo può motivarsi secondo circostanze specifiche (il nome del padrone, motivazioni occasionali, ecc. $)^{8}$.

\footnotetext{
5 Cfr. Veronelli (1971: 99).

"Il nostro mondo" (https://bibite.sanpellegrino.it/bibite/cocktail; consultato il 7 luglio 2017).

7 San Pellegrino bitter $>$ Sanbitter con caduta di pellegrino dall'elemento centrale, concrezione. Secondo noi parte della fortuna della campagna pubblicitaria è attribuibile agli aspetti fonetici legati al nome. Infatti, un'inventata pronuncia ossitona del nome consentiva un gioco di parole nello slogan "Sanbitter c'est plus facile!" (cfr. https://www.leonardoromanelli.it/sanbitter-cest-plus-facile/, consultato il 20 agosto 2017; lo spot è ancora disponibile su youtube: https://www.youtube.com/watch?v=xPI4VHXHb04, consultato il 20 agosto 2017).

8 È il caso dell'amaro San Carlo di Costigliole d’Asti, ispirato al nome (Carlo) del figlio
} 


\section{Denominazione di origine toponimica}

\subsection{Toponimi propriamente detti}

Alcuni di questi liquori o amari, sono da ricondurre al toponimo d'origine, come l'“Elisir San Marzano" o l'“Amaro San Severino" rispettivamente dalle località di San Marzano o San Severino.

Riteniamo che in questi casi non si tratti di sacro o di profano, ma semplicemente di indicazione di origine, non più indicativi o significativi dei pomodori San Marzano.

Si può invece osservare come storicamente in Piemonte, la provenienza del vino fosse spesso l'unica nominazione utilizzata, dal momento che tradizionalmente il vino era bianco o rosso (in Piemonte "nero") e ciò anche presso le mense dei sovrani. A titolo di esempio si citano alcuni conti relativi agli acquisti della Real Casa: nel 1785 venne acquistato del "vino di S. Giorgio" sullo stesso piano del "vino di Nizza bianco"10 o del "vino di Borgogna"

Potremmo affermare che in questo caso il destino del vino sia quello dell'acqua minerale o delle fonti che hanno "nomi santi": "San Bernardo", "Sant’Anna" (di Vinadio), "Sant'Angelo" molto diffuso in meridione ${ }^{12}$, ecc.

$\mathrm{Si}$ può inoltre considerare la nominazione attraverso un toponimo come una tendenza in espansione guidata da motivazioni economiche; sono note a tutti le campagne di stampa e le normative volte a valorizzare i prodotti gastronomici tipici di un territorio. Se poi il luogo ha il nome di un santo si colora di elementi che possono solo aiutare.

In direzione opposta, la località può indicare l'origine e rafforzare il senso di esotico, come nel caso dei cocktail, bevande moderne per eccellenza ${ }^{13}$. Così per il cocktail San Francisco, è immediata la relazione tra una bevanda che costituisce di per sé un richiamo al godimento e al lusso holliwoodiani e una città americana simbolo di vita godereccia e gozzoviglie da celebrità.

Più in generale a proposito dei cocktail e, sempre sull'onda della sorpresa e un po' della trasgressione, alcuni di questi hanno nomi profani se non deliberatamente

del proprietario. Niente a che vedere con i santi, quindi (http://www.saperebere.com/amari-ebitter/amari-s/, consultato il 2 luglio 2017).

9 AST, Sezioni Riunite, Real Casa, Azienda della Casa di Sua Maestà, Relazioni a S.M., Relazioni di suppliche a S.M., reg. 24, 1785, p. 71 (sotto la voce «vino di riforma»).

10 AST, Sezioni Riunite, Real Casa, Azienda della Casa di Sua Maestà, Relazioni a S.M., Relazioni di suppliche a S.M. reg. 24, 1785, p. 114.

11 AST, Sezioni Riunite, Real Casa, Azienda della Casa di Sua Maestà, Relazioni a S.M., Relazioni di suppliche a S.M. reg. 25, 1786, p. 119.

12 Per un repertorio delle fonti meridionali cfr. Germani (2015: 684).

13 I siti relativi ai cocktail sono molto numerosi, ne citiamo alcuni che sono stati utili al presente lavoro. Un sito con un elenco alfabetico che ne comprende molti,:http://www.azcocktails. it/ricetta_cocktail/500/san_francisco_analcolico.html (consultato il 7 luglio 2017); il sito ufficiale che fa riferimento all'IBA (International Bartenders Association): http://iba-world. com/iba-cocktails/ (consultato il 7 luglio 2017). 
irreligiosi, come il venezuelano "El Infierno"14 o "Il peccato di Eva" ${ }^{15} \mathrm{o}$ il "Sangue del diavolo" ${ }^{\prime 6}$. Certo più ispirato all'amore profano che ad un santo il cocktail "San Valentino". Si tratta evidentemente di fenomeni di moda volti a stupire, ma alcuni hanno radici un po' più lontane nel tempo e una sorta di tradizione sancita dal cinema americano, luogo per eccellenza di celebrazione del cocktail. "El Diablo", ad esempio, risale agli anni Trenta e compare nel film "L'impareggiabile Godfrei" (1936) $)^{17}$.

\subsection{Toponimi relativi all'abbazia di produzione/ santo patrono al quale si intitola un'abbazia}

Si tratta dell'eventualità più interessante dal punto di vista storico quella in cui la bevanda alcolica faccia riferimento all'abbazia o al convento di religiosi dove il liquore è nato.

In questo caso ci si trova in una tradizione differente ispirata non al vizio ma alla virtù terapeutica o taumaturgica ${ }^{18}$.

Riteniamo che l'ambito di sviluppo di questa tipologia onomastica dipenda dalla duplice natura delle bevande alcoliche che tradizionalmente dal medioevo fungevano sia da nutrimento che da elisir terapeutico. Pietro da Bairo (1602:260) esaltava le virtù de vino "Il vino giova al corpo se bee con un poco di acqua perche nutrisce e può custodir la sanità del corpo" ${ }^{\prime 19}$. Per la cura della peste un medico piemontese raccomandava:

Il vino sij bianco, di grato odore, di sotil sostanza, di color d'oro, più tosto garbetto, che dolce, ò rosso, maturo e mediocre, come sono quelli delle nostre colline di Cavaglià, Saluciola, Azeglio. [...]. Il grosso, dolce, nero, e da fuggire, massime da quelli, che gia sono appestati, se non fossero in estrema debolezza, e li altri ristori non giovassero $[\ldots]$ (Alessandri 1586: 52v-53r).

L'elemento medicamentoso e miracolistico unisce proprio i due aspetti della strategia onomastica.

Gli elisir delle abbazie erano spesso composti di erbe e molte di queste hanno un nome popolare che fa riferimento a un santo: in Romagna l'"erba d'san Roc" èl'"achillea

14 Ma si trova anche in italiano il cocktail "Inferno" (www.1001 cocktails.com, consultato il 20 agosto 2017).

15 www.cucinaremeglio.it/ricetta-peccato_di_eva.html, consultato il 20 agosto 2017.

16 www.azcocktails.it/classificazione/2/4/pre-dinner.html, consultato il 7 luglio 2017.

17 Per la regia di Henry Koster con David Niven, June Allison, Martha Hyer e Eva Gabor (cfr. Hamilton 2010: 76).

18 In questo caso la relazione con l'acqua minerale non è da escludere. Anche le acque possono avere una tradizione curativa. Citiamo, a mero titolo di esempio, alle acque di S. Vincenzo descritte in una memoria del 1840 dal Dr. Giacinto Sachero professore di clinica medica all'Università di Torino “[...] la salutar sorgente venne scoperta nel 1770 dall'abate Perret, dotto sacerdote di S. Vincenzo" (Sachero 1840: 262).

19 Si è riprodotto fedelmente lo scritto anche nell'ortografia che non corrisponde a quella attuale (ciò anche per le citazioni successive). 
millefolium” dalle proprietà emostatiche ${ }^{20}$. Inoltre alcuni santi danno il nome a frutti o fiori dalla cui macerazione si può produrre un elisir medicamentoso, pensiamo, ad esempio, alla fava di Sant' Ignazio ${ }^{21}$; in Piemonte non si può non ricordare San Martino che, oltre a dare il nome alla rinomata pera "martin-sec", offre il suo nome al "martinet" "grappoli lasciati sulla vite che maturano più tardi"22. Ciò non riguarda naturalmente solo l'Italia: ricordiamo a mero titolo di esempio S. Roberto, patrono contro la resipola, che dà nome al "geranium robertianum" in passato officinale $e^{23}$.

Anche alcuni farmaci, in effetti, avevano nomi collegati al sacro. In un prontuario di medicinali conservato presso l'Archivio di Stato di Torino uno dei medicamenti è il "legno santo"24; questo rimedio sembra bilanciato da un più profano o pagano "lacrima dragone" 25 .

Dalla produzione di un liquore tradizionale medicamentoso diventa quindi abbastanza naturale che la sede dell'abbazia dia il nome al liquore. Così nel caso dell'"Amaro di Santa Maria al Monte" del convento dei Padri Reverendissimi di Santa Maria al Monte.

Si verifica altresì il caso in cui invece del toponimo di provenienza del liquore il riferimento sia al patrono dell'ordine. Risulta più opaco ai giorni nostri ma non certo in passato dove la familiarità con i santi era sicuramente maggiore.

\footnotetext{
20 Cfr. Migliorini (1968: 134).
}

21 Si tratta di un rampicante (delle Filippine e della Cocincina) della famiglia delle Loganiacee. "Questi, per quanto abbiano ricevuto dai gesuiti, che per primi ne introdussero l'uso in medicina, il nome di fave di Sant'Ignazio, non somigliano affatto a fave, ma hanno una forma prismatica irregolare; sono esternamente di color bruno pallido; l'albume è duro, corneo, nerastro. Si usano in medicina come purgante ed emetico, sono inodori e di sapore amarissimo, e contengono molta stricnina." (Cortesi 1932, ora disponibile on-line: http://www.treccani.it/enciclopedia/ fava-di-sant-ignazio_\%28Enciclopedia-Italiana\%29/, consultato il 20 agosto 2017). A quale sant'Ignazio si riferisca il frutto esotico non è immediato. Normalmente ci si riferisce a Sant'Ignazio di Loyola, fondatore dei gesuiti e probabilmente il frutto è a lui dedicato. Un altro S. Ignazio era soprannominato "Il Taumaturgo" e si tratta di Sant'Ignazio di Rostov (XIII secolo). Sant'Ignazio di Antiochia, martire sotto Traiano è invece invocato contro il mal di gola e la tigna (cfr. NPI, s.v. Ignazio).

22 Migliorini (1968: 133).

23 Migliorini (1968: 134).

24 AST (Archivio di Stato di Torino), Sezioni Riunite, Camera dei Conti, Piemonte, Art. 478, Atti di visita alle botteghe degli speziali, mazzo unico (visita del 1587). Sinonimo di legno santo era l'Albero di Sant'Andrea, ovvero, il "Diospyros Lotus" ("Pianta della famiglia delle ebenacee (Diospyros lotus), nota anche con i nomi di 'legno santo, loto falso, loto d'Egitto, guaiaco falso'; originaria dell'Asia, ha foglie verdi scure, fiori piccoli, isolati, e frutti a bacca globosa, giallorossiccia, mangerecci; si coltiva in Europa per ornamento e quale portainnesto per le piante di cachi; il legno, assai duro, si presta a svariati usi” www.treccani.it/ vocabolario/albero-di-santandrea/, consultato il 20 agosto 2017). Inutile dire che con l'Albero di Sant'Andrea si produce un liquore estivo (cfr. www. grappa.com/ita/le-grappe-aromatizzate/un-liquore-estivo_24/, consultato il 20 agosto 2017).

25 AST (Archivio di Stato di Torino), Sezioni Riunite, Camera dei Conti, Piemonte, Art. 478, Atti di visita alle botteghe degli speziali, mazzo unico (visita del 1587). 
I Cistercensi, ad esempio, preparavano pozioni ed elisir per la cura dei malati. Ecco quindi che 1'"Elixir San Bernardo" al rabarbaro dell'abbazia Casamari ${ }^{26}$ in provincia di Frosinone si inserisce in questa tradizione. Il riferimento a San Bernardo in questo caso è legato all'ordine. Ricordiamo, infatti, che San Bernardo di Chiaravalle (1090-1153) è patrono dei cistercensi e protettore degli apicultori ${ }^{27}$.

Le tradizioni possono essere difficili da ricostruire e, seppure infondate o inventate, vengono elaborate a ragion veduta. Due casi ci paiono eloquenti per la loro somiglianza, uno da Bassano del Grappa e uno torinese.

Il primo caso riguarda l'“Amaro San Giuseppe” e il "Liquore San Giuseppe”. Secondo quanto suggerisce il sito del produttore, da un lato giocano un ruolo i padri di San Giuseppe che occupavano il luogo ora dell'azienda. Dall'altro lato l'opera di un farmacista che aveva elaborato una ricetta ${ }^{28}$.

Il torinese "Amaro San Simone" prese nome dalla chiesa di San Simone e Giuda Taddeo a Torino. Trasferiti i padri, sul sito si stabilì un'osteria detta San Simone, lo stabile (tra l'attuale via Garibaldi e via San Francesco d'Assisi) fu sempre denominato San Simone e vi si stabilì anche una farmacia San Simone ${ }^{29}$. I nuovi produttori entrati in possesso dell'antica ricetta del liquore decisero di adottare l'antico nome ${ }^{30}$.

26 www.casamari.it/3/index.php/1-abbazia-di-casamari/18-la-liquoreria?jjj= 1503260924221 , consultato il 10/8/2017.

27 Cfr. NPI, s.v. Bernardo.

28 "Fin dal 1928 una delizia per il palato, frutto della ricerca e della passione erboristica di un farmacista, del quale il passare del tempo ha scolorito i connotati, che donò agli amici gesuiti la ricetta segreta di un liquore chiamato 'Amaro San Giuseppe' visto che a produrlo erano i religiosi dell'omonima Villa a Bassano del Grappa." (www.amarosangiuseppe.Com/AMARO_SAN_GIUSEPPE, REGOLARE_NATURALMENTE_e_LIQUORE_SAN_GIUSEPPE_DIGESTIVO_E_BASTA/ Amaro San Giuseppe.html, consultato il 23 agosto 2017). E ancora: "Dalla rivisitazione dell'antica ricetta dell'Amaro San Giuseppe e dal frutto quasi centenario di una tradizione che prosegue ininterrottamente dal 1928, nasce il nuovo LIQUORE SAN GIUSEPPE digestivo... e basta! Ispirato ad un vecchio manoscritto dimenticato nel fondo di un cassetto e abbandonato allo scorrere del tempo, ha origine un prodotto bassanese, artigianale, naturale, amabile, adatto a qualsiasi situazione." (www.amarosangiuseppe.com/ AMARO_SAN_GIUSEPPE,_REGOLARE_NATURALMENTE_e_LIQUORE_SAN_GIUSEPPE_DIGESTIVO_E_BASTA/Liquore_San_Giuseppe.html, consultato il 23 agosto 2017). Il messaggio rimbalza immediatamente sul web: "La ricetta originale era patrimonio di un farmacista ed erborista, le cui origini non sono conosciute, che donò ai frati la ricetta affinché potessero produrla. Realizzato l'amaro i Gesuiti gli diedero il nome del santo simbolo della dedizione al lavoro, silenzioso artefice del disegno divino" (http://www.saperebere.com/amari-e-bitter/amari-s/, consultato il 23 agosto 2017). In verità il legame onomastico con il santo marito di Maria e patrono di operai e artigiani è piuttosto debole (cfr. NPI, s.v. Giuseppe).

29 È spesso molto difficile individuare con certezza gli spostamenti degli edifici religiosi. Le guide torinesi d'epoca sono ambigue e situano la chiesa dei santi Simone e Giuda Taddeo in luoghi diversi: Craveri (1753: 165) "Seguitando la strada pel lungo Borgo del Pallone vedesi la Parrocchia, e Chiesa de' Ss. Appostoli Simone e Giuda. Sottoposta a' Canonici della Metropolitana”. L'isola di San Simone è menzionata senza presenza di chiese in una guida del 1801 (Breve descrizione 1801: 39).

30 Almeno così si afferma Cinzia Gatti in un articolo pubblicato sul web (http://www. 
Che le due tradizioni siano vere o inventate evidentemente il nome del santo è proficuo per vendere l'amaro e se vi è il legame con un ente religioso meglio ancora. In questo senso è imprescindibile il legame con le abbazie, luogo dove si preparavano spesso rimedi.

Il fenomeno è in decisa espansione. Dal momento che i prodotti enogastronomici godono di popolarità crescente i nomi di luoghi sacri vengono utilizzati per prodotti privi di tradizione. È il caso della birra San Michele prodotta dal birrificio omonimo in località Sant'Ambrogio non lontano dalla Sacra di San Michele uno dei monumenti più noti in Piemonte. Il sito del produttore confessa la data di nascita della sua birra nel $2010^{31}$, decisamente recente. Lo stesso sito reclamizza un antico quanto anonimo "Elisir dell'Abate", conosciuto come Ippocrasso "prodotto secondo la millenaria ricetta medioevale, tramandata dalle abbazie della Valle di Susa, dal delicato sapore e dalle apprezzate virtù digestive" 32 .

Anche la notissima Abbazia piemontese della Novalesa, nota dal punto di vista artistico e culturale, dispone di uno shop on-line dove si reclamizzano ogni sorta di prodotti più o meno medicamentosi ("Aloe del monaco") e liquori con un costante riferimento non tanto a santi quanto ai monaci ("Amaro del monaco", "Elisir dell'abate", "Liquirizia del monaco"33), certo confidando sulla diffusa fama di laboriosità e moderazione dei religiosi.

C'è un'interessante alternanza tra nome generico legato alla funzione e nome specifico. In effetti, i santi hanno una sorta di competenza rispetto alle virtù taumaturgiche. Risulta quindi più facile attribuire un nome generico che legare l'elisir a una personalità specifica.

Dalle abbazie si può attuare un riferimento legato in senso proprio alle capacità e virtù di un santo.

\section{Nomi di liquori legati alle virtù di un santo ma non ad un luogo}

Il caso del piemontese "Nocino di San Giovanni” è particolare. Non sono disponibili molte informazioni attendibili che attestino l'antichità del liquore, ma i pubblicisti hanno subito cavalcato l'onda della popolarità del santo legato al giorno del

torinoggi.it/2016/09/17/leggi-notizia/argomenti/eventi-11/articolo/sotto-via-garibaldile-origini-dellamaro-san-simone.html, consultato il 2 luglio 2017) che racconta anche del ritrovamento della targa di una farmacia, la farmacia Tacconi. Si aggiunga che la Guida Paravia (1936-37: 1440) menziona una farmacia "San Simone" in via Garibaldi 13 il cui titolare era il dr. Tacconis. In ogni caso abbonda il materiale su internet che riguarda questo amaro con, grosso modo, gli stessi contenuti, tra la farmacia e dei padri non sempre specificati: http://www. amarosansimone.com/amaro-san-simone.html?fname=1969, consultato il 2 luglio 2017 e https: / / torino.diariodelweb.it/ torino/ articolo/?nid=20170323_408072, consultato il 2 luglio 2017.

31 Cfr. http://www.prolocosantambrogio-sacrasanmichele.it/?p=832 (consultato il 16 luglio 2017).

$32 \mathrm{http}: / /$ www.prolocosantambrogio-sacrasanmichele.it/?p=832 (consultato il 16 luglio 2017).

33 http://www.abbazianovalesa.org/aliment3liquori.htm (consultato il 20 agosto 2017). 
patrono (24 giugno). Su alcuni siti internet si lega questa giornata alla raccolta delle noci (ma la forzatura stagionale per il Piemonte è evidente!) e ai raduni delle streghe: il legame tra sacro e profano è immediato ${ }^{34}$. In verità a san Giovanni Battista si ispira la raccolta di altre erbe tra le quali la verbena. Infatti, la "tradizione magica riconosce alla notte di S. Giovanni, così prossima al solstizio d'estate, un momento particolarmente proficuo per diversi riti d'origine pagana, adatto, tra l'altro, a raccogliere erbe per preparare pozioni taumaturgiche" 35 .

San Giovanni Evangelista, invece, è patrono dei vignaioli e nel giorno della sua festa (27 dicembre) si benedice il vino "si riteneva inoltre che chi lo venerava fosse immune da avvelenamenti e che potesse bere vino senza danni" ${ }^{36}$.

Un altro caso è l'"Amaro San Genesio", prodotto in Piemonte in provincia di Asti, offerto da una compagnia teatrale alla fine dello spettacolo (siamo alla fine dell'Ottocento) per rimpinguare i magri guadagni ${ }^{37}$. Evidentemente l'operazione funzionò tanto da battezzare il liquore con il nome del protettore degli artisti. San Genesio, infatti, martire al tempo di Diocleziano era un mimo che mentre sbeffeggiava i cristiani fu graziato e chiese di essere battezzato, ottenendo al contempo il martirio ${ }^{38}$. Se anche la tradizione di questo amaro è stata inventata, la creatività si è sviluppata in modo coerente con la tradizione.

In conclusione possiamo affermare che quello che potrebbe sembrare un terreno di studio piuttosto semplice ed uniforme ad un'analisi più attenta si rivela molto più complesso ed articolato. La tradizione storica non è l'unica spiegazione del quadro onomastico; al contrario, il legame con i santi si trasforma in un fattore di marketing in una società secolarizzata.

\section{Bibliografia}

Alessandri, F. 1586. Trattato della peste et febri pestilenti. Torino: Antonio de' Bianchi. ASTo, Art. 478 = ASTo (Archivio di Stato di Torino), Sezioni Riunite, Camera dei Conti, Piemonte, Art. 478, Atti di visita alle botteghe degli speziali, mazzo unico.

34 È quello che afferma Manuela Zanni in un articolo su internet: "Lalbero di noci e i suoi frutti viene spesso associato all'idea di riti ed incantesimi. Ci sono parecchie leggende, infatti, che designano il noce come albero delle streghe, perché si crede che ne utilizzino i rami per volare e che organizzino i loro sabba proprio intorno al noce, nella notte tra il 23 e il 24, raccogliendone i frutti verdi per i loro rituali, e garantendo così vitalità alla pianta proprio per il fatto che il tutto avviene nella notte più corta, quando la luce vince sulle tenebre." (www.idraincoming. wordpress.com, consultato il 3 luglio 2017). E in un titolo: "Il nocino di San Giovanni: un liquore antico a metà tra sacro e profano”. Pubblicato il 23 giugno 2016 (http://www.saggieassaggi. it/il-nocino-di-san-giovanni-un-liquore-antico-a-meta-tra-sacro-e-profano/, consultato il 3 luglio 2017).

35 NPI, s.v. Giovanbattista.

36 NPI, s.v. Giovanni.

37 La storia è raccontata sul sito http://www.saperebere.com/amari-e-bitter/amari-s/ (consultato il 2 luglio 2017).

38 Cfr. MPI, s.v. Genesio. 
Bairo, P. 1602. Secreti medicinali. Venezia: Nicolo Tebaldini.

Bertini Malgarini, P., U. Vignuzzi. 2003. Un ignoto volgarizzamento umbro del 'Liber Specialis Gratiae' di Matilde di Hackerborn. In Filosofia in volgare nel Medioevo, vol. 1, N. Blay e L. Sturlese (eds.), 419-432. Louvain la Neuve: Fédération Internationale des Instituts d'Etudes Médiévales.

Breve descrizione della Comune di Torino nell'anno IX repubblicano con i nomi dé possessori delle case i numeri dell'isole e di quelli che indicano le abitazioni. 1801. Torino: Ferrero e Pomba.

Comba, R. 1990. Vigne e vini nel Piemonte medievale. Cuneo: L'Arciere.

Cortesi, F. 1932. Fava di Sant'Ignazio. In Enciclopedia Italiana. http://www.treccani.it/enciclopedia/fava-di-sant-ignazio_\%28Enciclopedia-Italiana\%29/ (consultato il 20 agosto 2017).

Craveri, G.G. 1753. Guida dei forestieri per la real città di Torino. Torino: Rameletti.

Croce, G. B. 1606. Della eccellenza e diversità dei vini. Che nella montagna di Torino si fanno; E del modo di farli. Torino: Pizzamiglio.

De Groff, D. 2008. The Essential Cocktail. The Art of Mixing Perfect Drinks. New York: Clarkson Potter/Publishers.

DEI: Battisti, C., G. Alessio. 1957. Dizionario Etimologico Italiano. Firenze: Barbèra.

DELIN: Cortellazzo, M., M. Cortellazzo. 1999 [1989]. Il nuovo etimologico. DELI - Dizionario etimologico della lingua italiana. Bologna: Zanichelli.

DSA: Di Sant’Albino, V. 1859. Gran Dizionario Piemontese-Italiano. Torino: L'Unione Tipografico-Editrice.

Ernst, G. 1966. Un ricettario di medicina popolare in romanesco del Quattrocento. SLI VI: $138-163$.

Felecan, O., N. Felecan. 2016. Denumiri ale băuturilor spirtoase tradiționale româneşti. Dacoromania XXI (1): 46-59.

Gatta, F. L. 1833. Cenno intorno alle viti e ai vini della provincia d'Ivrea. In Calendario georgico della Reale Società Agraria di Torino per l'anno 1833. Torino: Tipografia Chirio e Mina.

Gentile, S. 1994. Le piante negli scambi fra Americhe ed Europa (Italia in particolare). In L'età delle scoperte geografiche nei suoi riflessi linguistici in Italia, 217- 227. Firenze: Accademia della Crusca

Germani, A. 2015. Denominazioni delle sorgenti dell'Italia meridionale (parte II). In Name and Naming. Conventional/Unconventional in Onomastics, O Felecan (ed.), 680-690. Cluj: Editura Mega- Editura Argonaut.

Guida di Torino. 1937-1938. Torino: Paravia.

Hamilton, O. 2010. Star cocktails. Tutti i cocktail dei classici del cinema. Milano: Baldini e Castoldi

Lauro, P. 1558. Libro delle quattro infermità cortigiane, che sono Catarro, Gotta, Artetica, Sciatica: Mal di Pietre, \& di Reni: Dolore di Fianchi, et Mal Francese, \& altre cose utilissime. Venezia: Giovanni Battista \& Marchio Seffa.

Lerda, A. 1978. Il "Vademecum per il popolo" di un medico piemontese del '500. Studi Piemontesi VII (1): 113-117. Torino: Centro Studi Piemontesi.

Levi, A. 1927. Dizionario etimologico piemontese. Torino: Paravia.

Migliorini, B. 1968. Dal nome proprio al nome comune. Firenze: Olschki (ristampa fotostatica dell'edizione del 1927 con un supplemento).

Nada Patrone, A. M. 1982. Gli uomini e le loro malattie nel tardo Medio Evo (da tre testi medici pedemontani). Studi Piemontesi XI (1): 68-82. Torino: Centro Studi Piemontesi.

Neethling, B. 2017. The Role of Anthroponymic Commemoration on Wine Labels in South Africa. Names 20 (20). 
NPI: Rossebastiano A., E. Papa (eds.) 2005. I nomi di persona in Italia. Dizionario storico ed etimologico. UTET: Torino.

Nuessel, F. 2010. A Note on Names for Energy Drink Brands and Products. Names 58 (2): $102-110$.

Pipino, M. 1758. Vocabolario piemontese. Torino: Stamperia Reale.

REP: Cornagliotti, A. (ed.) 2015. Repertorio Etimologico Piemontese. Torino: Centro Studi Piemontesi.

Rohlfs, G. 1966-1969. Grammatica storica della lingua italiana e dei suoi dialetti. Torino: Einaudi, 3 voll.

Rossebastiano, A. 2009. Mestieri veri e falsi tra vigne, cantine e taverne del Piemonte medievale. In Mestieri, soprannomi e altra onomastica, D. Cacia, A. Perinetti, C. Colli Tibaldi (eds.), 53-68. Alessandria: Edizioni dell'Orso.

Rossebastiano, A. 2010. Soprannomi medievali piemontesi tra ironia ed insulto. In I soprannomi nell'antroponimia, E. Papa (ed.), 31-46. Alessandria: Edizioni dell'Orso.

Rossebastiano, A. 2011. La coltivazione della vite nell'onomastica del Piemonte. In Saggi di toponomastica, E. Papa, D. Cacia (eds.), 3-17. Alessandria: Edizioni dell'Orso.

Rossebastiano, A. 2015. Memorie di Spagna sotto la Mole. In Hora fecunda. Scritti in onore di Giancarlo Depretis, P. Calef, F. Estévez, A. Fournier (eds.), 307-333. Torino: Nuova Trauben.

Sacero, G., 1840. Memorie originali. Sulle acque minerali aciduolo-saline di S. Vincenzo nella valle d'Aosta. In Giornale delle scienze mediche, anno 3, Vol. 7, 257-306. Torino: Alessandro Fontana.

Serianni, L. 1989a. Grammatica italiana. Torino: UTET.

Serianni, L. 1989b. Lingua medica e lessicografia specializzata nel primo Ottocento. In Saggi di storia linguistica italiana, 77-139. Napoli: Morano Editore.

Serianni, L. 2005. Un treno di sintomi. Milano: Garzanti.

Serianni, L. 2011. Manuale di linguistica italiana. Storia, attualità, grammatica. Milano: Bruno Mondadori

TLIO: Beltrami P. G. (ed.). 1998ss. Tesoro della lingua italiana delle origini. Firenze. http://tlio. ovi.cnr.it/ TLIO/ (consultato il 20 agosto 2017).

Veronelli, L. 1971. Il vino giusto. Milano: Rizzoli.

Vopisco, M. 1564. Promptuarium. Mondovì: Torrentino (ristampa anastatica a cura di G. Gasca Queirazza, Torino: Bottega d'Erasmo, 1972).

Zalli, C. 1830 [1815]. Dizionario piemontese, italiano, latino e francese. Carmagnola: Tipografia di Pietro Barbié. 